\title{
Photoinduced collective motion of oil droplets and concurrent pattern formation in surfactant solution
}

Tomoya Kojima, Hiroyuki Kitahata, Kouichi Asakura, and Taisuke Banno*

["] Tomoya Kojima, Prof. Kouichi Asakura Dr. Taisuke Banno

Department of Applied Chemistry, Faculty of Science and Technology, Keio University, 3-141 Hiyoshi, Kohoku-ku, Yokohama, 223-8522, Japan

E-mail: banno@applc.keio.ac.jp

Prof. Hiroyuki Kitahata

Department of Physics, Graduate School of Science, Chiba University, 1-33 Yayoi-cho, Inage-ku, Chiba 263-8522, Japan

\begin{abstract}
Collective motion is ubiquitous in living systems. Although various biomimetic artificial systems have been constructed, there have been few studies reported on collective motion induced by the coupling of chemical reactions, diffusion and convection in a far-fromequilibrium state. In this study, we report an artificial system of oil droplets in a surfactant solution wherein the collective motion of multiple droplets and pattern formation occurred concurrently. Using photo-responsive surfactants with an azobenzene moiety, the assembly of droplets and the formation of circular patterns around the formed droplet clusters occurred under UV illumination, whereas the disassembly of droplets and disappearance of the patterns occurred under subsequent visible light illumination. The observed dynamics were induced by Marangoni flows based on the reversible photoisomerisation of azobenzenecontaining surfactants. The phenomena were considered analogous to the bioconvection of microorganisms. These findings could be useful for understanding the mechanism of motion of life in terms of physicochemical aspects.
\end{abstract}




\section{Introduction}

Motion is one of the essential dynamics for characterising life. To approach the essence of motion in living systems, microorganisms have been utilised as models. They respond to external stimuli and change their mode of motion. For example, the cellular slime mould Dictyostelium discoideum and the nematode Caenorhabditis elegans exhibit taxis in response to chemicals, ${ }^{[1]}$ light, ${ }^{[2]}$ electricity, ${ }^{[3]}$ and temperature. ${ }^{[4]}$ Alga Chlamydomonas ${ }^{[5]}$ and Euglena ${ }^{[6]}$ with flagella also show taxis to light irradiation, and the positive and negative modes are switchable depending on the intensity. Moreover, they generate bioconvection as collective behaviour owing to their individual phototaxis and the surrounding hydrodynamic effect, resulting in the formation of a macroscopic ordered pattern. ${ }^{[7]}$ Various reductive and constructive approaches have revealed that the characteristic dynamics of microorganisms are induced by hierarchical chemical reaction networks. ${ }^{[8]}$ However, the mechanisms have not yet been fully elucidated owing to their complexity. Therefore, the construction of simpler artificial systems that reproduce the characteristic behaviour of organisms would allow us to consider complicated living systems as physicochemical phenomena. ${ }^{[9]}$

Thus far, various artificial objects that transduce chemical energy from external stimuli to their own mechanical energy have been created. ${ }^{[10]}$ Solid anisotropic particles, that is, Janus particles, exhibit directional motion induced by light irradiation, ${ }^{[11]}$ electric field ${ }^{[12]}$, decomposition of $\mathrm{H}_{2} \mathrm{O}_{2},{ }^{[13]}$ and thermal gradient ${ }^{[14]}$. Liquid droplets perform programmed motion, including directional motion and simple tasks in response to light, ${ }^{[14]}$ temperature, ${ }^{[15]}$ $\mathrm{pH}^{[16]}$ salt concentration, ${ }^{[17]}$ and metal ions. ${ }^{[18]}$ In addition, the collective motion of solid particles without anisotropy emerged owing to electric-field-induced fluid flows ${ }^{[20]}$ and the diffusiophoresis of ions that are generated by the photodecomposition of metal particles ${ }^{[21]}$. Motion of droplets induced by the dissolution of droplets has also been demonstrated. ${ }^{[22]}$ Despite considerable progress made to date, no examples of more complicated biomimetic systems have been reported where the directional motion of objects induces other macroscopic dynamics, such as collective motion and pattern formation, triggered by a coupling of chemical reactions, diffusion and convection.

Thus, we herein report an artificial system wherein the convection of oil droplets exhibiting taxis is associated with their collective motion. The main driving force of moving liquid droplets in a surfactant solution is estimated to be Marangoni flows based on the 
heterogeneity in the interfacial tension at the droplet surface. ${ }^{[23]}$ To construct the targeted system, we focused on photo-responsive surfactants with an azobenzene moiety (AzoTAB, Fig. 1a) because the use of light as an external stimulus has the advantage of fine control of the position and area. Some studies have previously reported that photoisomerisation of azobenzene-containing surfactants between trans- and cis-isomers induces Marangoni flows due to local changes in the interfacial tension of the droplet surface, resulting in the taxis of droplets. ${ }^{[14 a, d]}$ Therefore, by using AzoTAB, we expected that specific convection around each oil droplet exhibiting taxis was generated, inducing the collective motion of multiple droplets.

\section{Results and Discussion}

The dynamics of the oil droplets in a surfactant solution under light illumination were observed. The observation sample was prepared by gently mixing $4.3 \mu \mathrm{L}$ of heptyloxybenzaldehyde (HBA) with $85.7 \mu \mathrm{L}$ of $50 \mathrm{mM}$ mixed solution, including azobenzenecontaining surfactant (AzoTAB) and dodecyltrimethylammonium bromide (DTAB) at the equivalent molar ratio (Figure 1a). The dispersions were poured into a pool-like sealed chamber (Figure 1b) and observed under a phase-contrast microscope. Droplets with diameters in the range of 5-170 $\mu \mathrm{m}$ (Figure S1) were observed, and their motion time when all droplets ceased their motion in the chamber was $13 \pm 3$ min on an average of 10 trials. Because the density of oil and the surfactant solution was 0.99 and nearly 1.0 respectively, droplets were in the vicinity of an upper cover slip in the chamber due to buoyancy when they ceased the motion. Next, we performed a light-illumination experiment for $180 \mathrm{~s}$ for each trial. When the dispersion was illuminated with UV light at $1.2 \mathrm{~mW} / \mathrm{cm}^{2}$ for $180 \mathrm{~s}$ from the upper side, droplets started to move to the lower side and gradually assembled with each other (Figure 1c and Supplementary Movie S1). Note that, immediately after UV illumination, the droplets moved slightly against UV in the lateral direction for a few seconds because the emulsion was not illuminated from the vertical upper side, but from the diagonal upper side. After assembly, circular patterns around each droplet cluster gradually appeared. Using hydrophobic and hydrophilic fluorescent dyes, 5-FITC, Nile Red, Rhodamine 6G, Rhodamine B, and Uranine, allowed them to accumulate in the patterns (Figure S2). The patterns were larger with the size of the clusters (Figure S3). Under the subsequent visible (vis) light illumination at $5.8 \mathrm{~mW} / \mathrm{cm}^{2}$, droplets in the clusters disassembled, and the circular patterns 
disappeared (Figure 1c and Supplementary Movie S2). We considered a series of assembly and disassembly dynamics of droplets under light illumination as the collective motion coupled with phototaxis. The time course of the height of the oil droplets was observed (Figure S4). The oil droplets first moved downward under UV illumination. Smaller droplets tended to rapidly move upward when they approached the other droplets and formed a cluster. After UV illumination for $180 \mathrm{~s}$, each droplet in a cluster was almost in contact with the upper cover slip (Figure S5 and Table S1). Under vis illumination, all the observed droplets did not change their height significantly and remained near the upper cover slip. The collective motion and pattern formation were observed repeatedly under UV illumination for $180 \mathrm{~s}$ and subsequent vis illumination for $180 \mathrm{~s}$, alternated three times (Figure S6). When the intensity of light was higher, the same phenomena were observed under UV illumination (Figure S7). In contrast, disassembly behaviour depended on the intensity of the vis light: the stronger the illumination, the more the oil droplets were dispersed (Figure S8). The dynamics were also influenced by the thickness of the chamber (Figure S9). Collective motion was clearly observed when the thickness was greater than $160 \mu \mathrm{m}$. These results suggest that the photoinduced dynamics depend on the experimental conditions. 
(a)

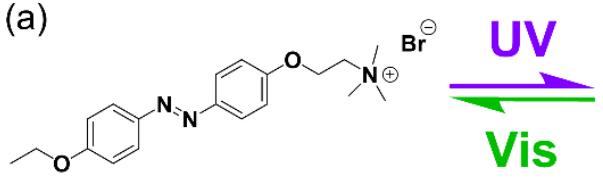

trans-AzoTAB

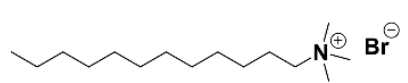

DTAB

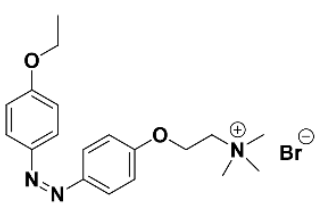

cis-AzoTAB

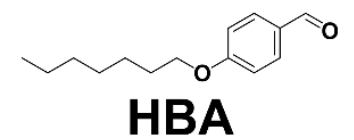

(b)

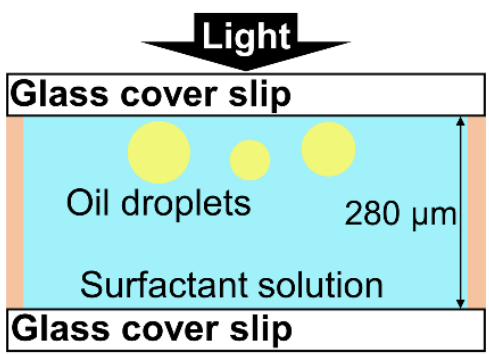

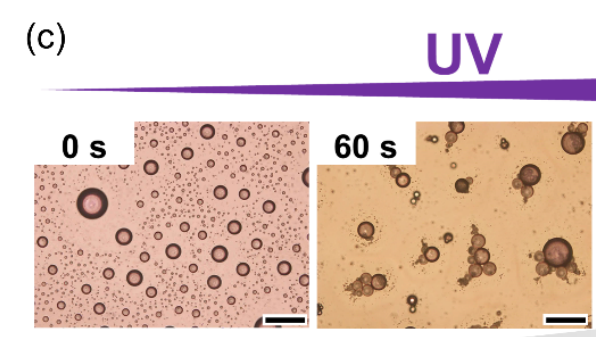

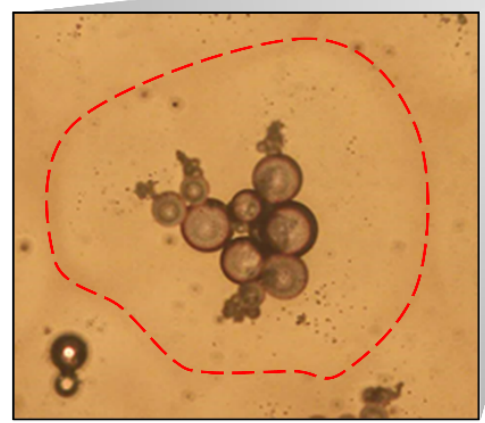

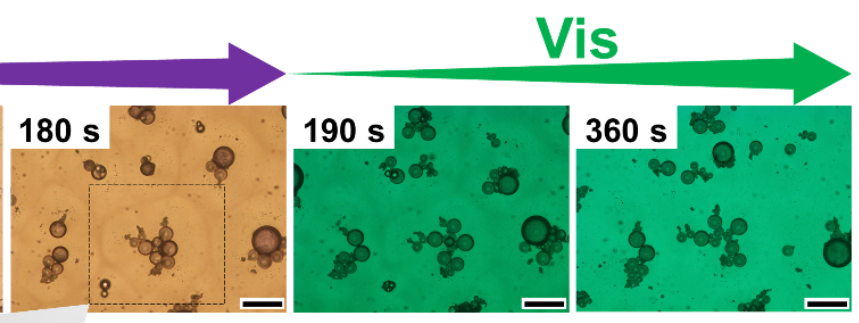

(d) 250

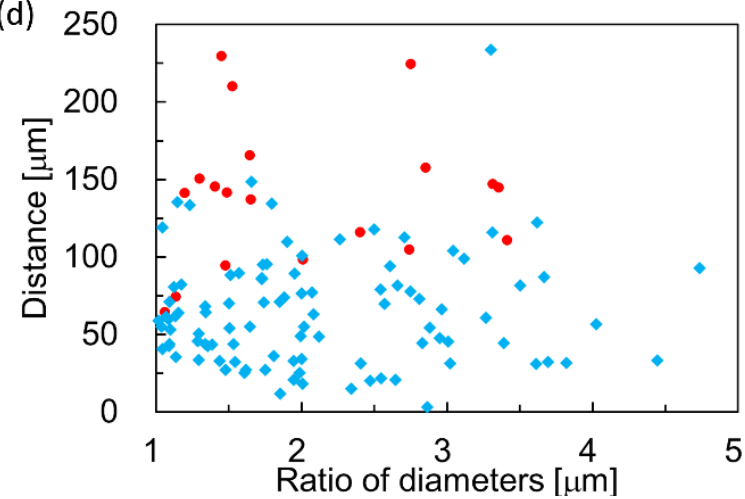

Figure 1. Collective motion of droplets and pattern formation. (a) Chemical structures of surfactants and oil. (b) A pool-like sealed chamber with the depth of $280 \mu \mathrm{m}$ used in the microscope observation. The chamber was illuminated with light from the upper side. (c) Typical sequential micrographs of HBA droplets in $50 \mathrm{mM}$ AzoTAB/DTAB (50/50 mol\%) solution under light illumination. Dotted red line in an enlarged micrograph represents one of the patterns. Scale bar: $200 \mu \mathrm{m}$. (d) Observation results of the initial distance between two droplets and the ratio of their diameters. Blue and red symbols indicate occurrence and no occurrence of assembly dynamics, respectively. 
Focusing on the assembling behaviour of oil droplets under UV illumination, the threshold of the distance was investigated. To exclude the influence of the lateral motion observed immediately after UV illumination, the distance between two droplets was determined after the droplets ceased lateral motion. From the relationship between the ratio of diameters and distance between two droplets, the threshold of distance for the occurrence of assembly was confirmed to be approximately $100 \mu \mathrm{m}$ regardless of the difference in the diameters. (Figure 1d). In the emulsion with a lower number density of droplets prepared using a smaller amount of HBA, a similar threshold was observed (Figure S10a). In addition, no difference was observed in the threshold under UV illumination with a higher intensity (Figure S10b). These results clearly indicate that the assembly of droplets under UV illumination depends on the distance between droplets.

To clarify the mechanism by which both the collective behaviour of droplets and pattern formation emerged, we first investigated the photoisomerisation of AzoTAB by UV-vis spectroscopy. A $50 \mathrm{mM}$ AzoTAB/DTAB (50/50 mol\%) solution without HBA which was illuminated with light was diluted and measured. The cis-isomer ratio was calculated using the calibration curve, which was based on the absorbance at $357 \mathrm{~nm}$, the maximum absorption wavelength of the trans-isomer (Figures S11a and b). Given that the half-life of AzoTAB from its cis- to trans-isomer was estimated to be $13 \mathrm{~h}$ (Figure S11c), spontaneous isomerisation was negligible under the tested conditions. The isomerisation from the transto cis-isomer gradually occurred under UV illumination, whereas reverse photoisomerisation occurred under vis illumination (Figure 2a). Further, the isomerisation rate increased as the intensity of the light increased (Figure S12). 
(a)

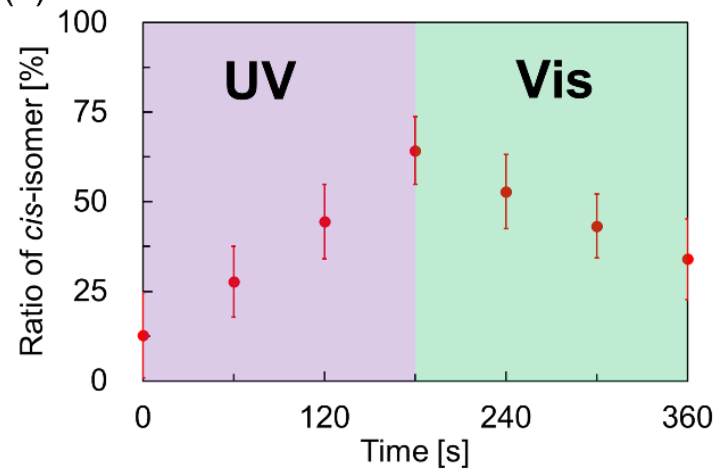

(c)

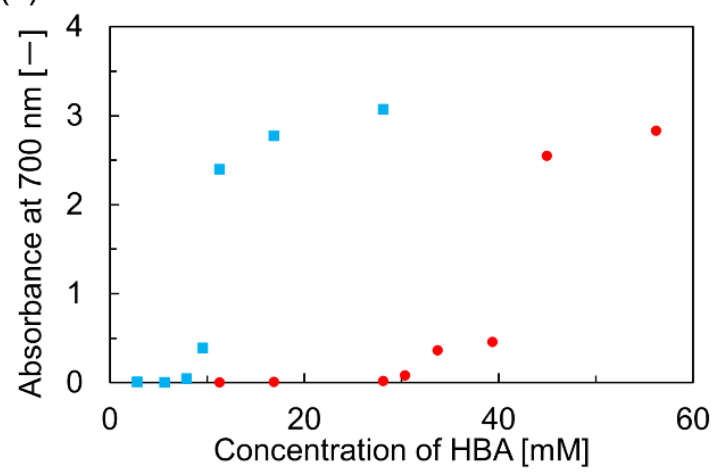

(b)

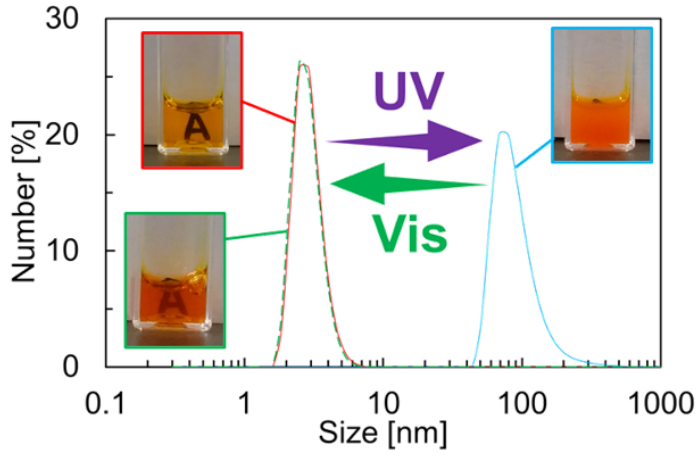

(d)

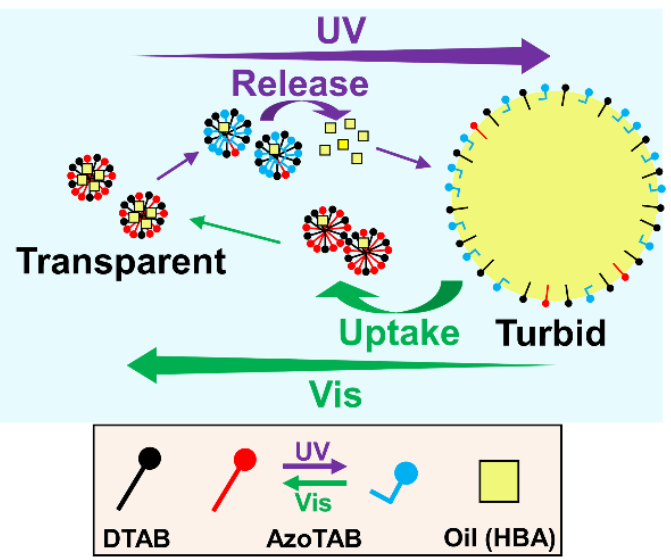

Figure 2. Surfactant properties of AzoTAB before and after light illumination. (a) Time-course of the isomerisation ratio of AzoTAB in $50 \mathrm{mM}$ AzoTAB/DTAB (50/50 mol\%) solution under UV and subsequent vis illumination. (b) Size distribution of nanoparticles of filtrates before (red) and after UV illumination (blue), and after subsequent vis illumination (dotted green) analysed by dynamic light scattering. Turbidity of filtrates observed by the naked eye is shown in insets. As the paper where the letter " $A$ " is written is placed behind the cell, the letter "A" can be viewed if the filtrate is transparent. (c) Relationship between absorbance at $700 \mathrm{~nm}$ and concentration of HBA per $50 \mathrm{mM}$ AzoTAB/DTAB (50/50 mol\%) solution when AzoTAB was trans-isomer rich (red) and cis-isomer rich (blue). (d) Schematic representation of change at a nanometre scale. 
Next, we examined the change in surfactant properties by the photoisomerisation of AzoTAB under UV and vis illumination. According to surface tension measurements by the pendant drop method, the critical micelle concentration (CMC) of AzoTAB and DTAB mixed in the equivalent molar ratio when AzoTAB was trans-isomer rich or cis-isomer rich was determined. Before the measurements, the cis-isomer ratio of AzoTAB in the cis-isomer-rich solution was confirmed to be more than $90 \%$. The CMC was $15 \mathrm{mM}$ and $16 \mathrm{mM}$ for the transisomer-rich and cis-isomer-rich solutions, respectively (Figure S13a). In addition, the diameter of the micelles in both the $50 \mathrm{mM}$ trans-AzoTAB-rich solution and cis-AzoTAB-rich solution was a few nanometres according to the dynamic light scattering (DLS) method (Figure S13b). We also measured the particle diameter in $50 \mathrm{mM}$ AzoTAB/DTAB (50/50 mol\%) solution containing HBA after filtration using a $0.8 \mu \mathrm{m}$ syringe filter. The DLS measurements showed that the diameters before and after UV illumination were $3 \mathrm{~nm}$ and $70 \mathrm{~nm}$, respectively (Figure $2 \mathrm{~b}$ ). Under subsequent vis illumination, the size distribution returned to its original value. This result was in accordance with observations of the filtrate by the naked eye (Figure $2 \mathrm{~b}$ insets). The filtrate was initially transparent, and it became turbid under UV illumination and then returned to transparent under subsequent vis illumination. To estimate the maximum amount of solubilised HBA in micelles that included much trans- or cis-AzoTAB, we measured the absorbance intensity at $700 \mathrm{~nm}$, where the peak from AzoTAB, DTAB, and HBA was not detected, as a barometer of turbidity at room temperature. The maximum solubilised amount was estimated to be the oil volume when the absorbance intensity increased, indicating that the mixture became turbid. The relative ratio of the solubilised amount into micelles was 3.6 to 1.0, as observed in Figure 2c. Based on these results, due to the photoisomerisation of AzoTAB, HBA was released from the micelles, and larger particles were generated under UV illumination. Meanwhile, HBA was resolubilised into the micelles under subsequent vis illumination (Figure 2d).

In addition, the effect of AzoTAB isomerisation on the interfacial tension between the AzoTAB/DTAB (50/50 mol\%) solution and HBA was investigated using the drop weight method. The interfacial tension of the solution containing a large amount of trans-isomer $(>90 \%)$ was higher than that containing a large amount of cis-isomer $(>90 \%)$ at the tested concentrations (Figure 3a). Therefore, it was considered that the interfacial tension between the oil and surfactant solution became higher or lower under UV or vis illumination, 
respectively, owing to the photoisomerisation of AzoTAB. Thus, we observed the interface between $50 \mathrm{mM}$ AzoTAB/DTAB (50/50 mol\%) solution and HBA in a glass vial when the specimen was illuminated with UV from the right side (Figures $3 b-c$ and Supplementary Movie S3). Convective flows from the left to the right side at the interface generated in each phase immediately after UV illumination. Because the interfacial tension increased owing to the photoisomerisation of AzoTAB from the trans- to cis-isomer, it was suggested that the interfacial tension of the illuminated side was higher than that of the non-illuminated side, inducing Marangoni flows from the area of lower interfacial tension to that of the higher one. Based on these results, it was clarified that the photoisomerisation of AzoTAB caused a difference in the interfacial tension that was sufficient for inducing Marangoni flows at the interface.

(a)

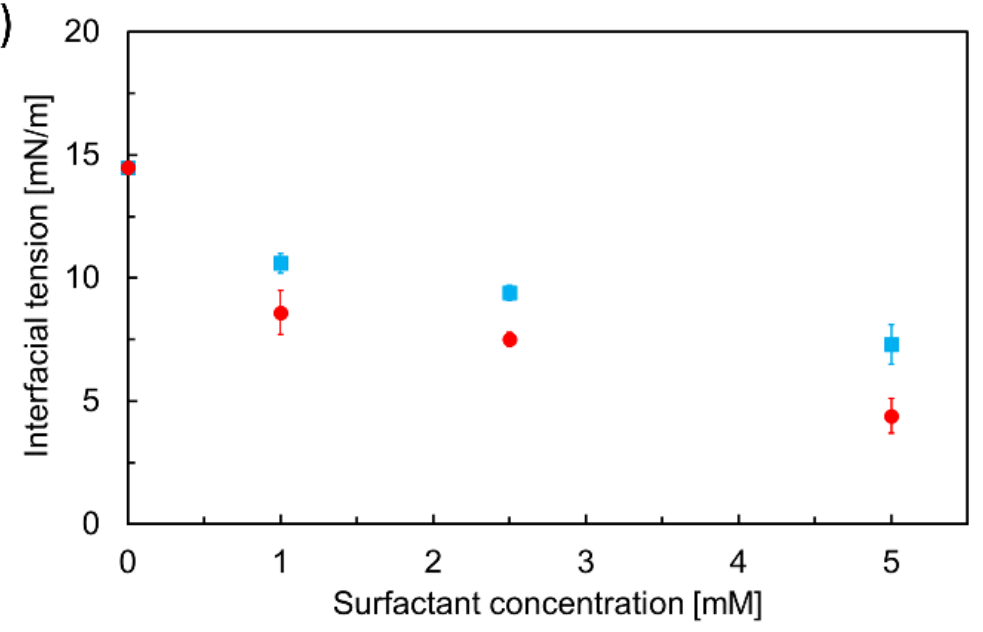

(b)

(c)
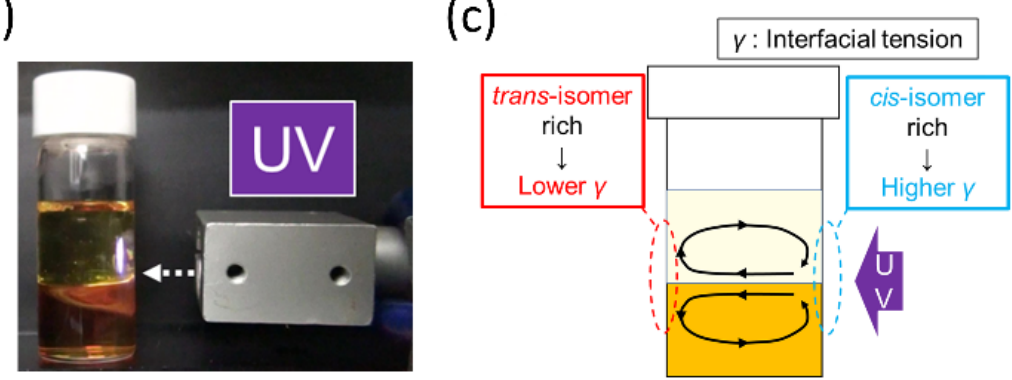

Figure 3. Variation in the interfacial tension along the photoisomerisation of AzoTAB. (a) Interfacial tension between AzoTAB/DTAB (50/50 mol\%) solution containing $>90 \%$ transisomer (red) or $>90 \%$ cis-isomer (blue) and HBA with the surfactant concentration according to the pendant drop method at room temperature. (b) Experimental setup of equipment. (c) Schematic representation of the observation of convective flows induced at the interface between $50 \mathrm{mM}$ surfactant solution (lower phase) and HBA (upper phase). 
Thus, we attempted to visualise the flow fields around the clusters of oil droplets induced by the photoisomerisation of AzoTAB using polyethylene fluorescent beads with a diameter of $10-22 \mu \mathrm{m}$. Because the density of the beads was $0.998 \mathrm{~g} / \mathrm{cm}^{3}$ and lower than that of the surfactant solution, which was nearly equal to $1.0 \mathrm{~g} / \mathrm{cm}^{3}$, the beads floated near the upper cover slip. It was observed that the beads moved radially from a droplet cluster and accumulated in line with circular patterns (Figures $4 a-b$ and Supplementary Movie S4). A few beads moved in the z-direction. The beads rose to the upper cover slip near the droplet cluster and went down in the area where the circular pattern was generated (Figure 4c and Supplementary Movie S5). This indicates that the variation of interfacial tension of the droplet surface by the photoisomerisation of AzoTAB induced convective flows between two areas where droplet clusters and circular patterns formed. Under vis illumination, the bead moved with or without circulating around a droplet cluster in the $x-z$ plane (Figure $4 d$ and Supplementary Movies S6 and S7). However, the observed motion was similar to that under UV illumination, especially with respect to the way of the circulation. The speed of the bead under both UV and vis illumination was $2-5 \mu \mathrm{m} / \mathrm{s}$, but rose temporarily to approximately 10 $\mu \mathrm{m} / \mathrm{s}$ when the bead was close to the droplet cluster (Figures S14-S16). Thus, we estimated the characteristic flow speed induced by droplet clusters from the speed of the fluorescent beads. The Reynolds number Re equals $\rho v L / \eta$, where $\rho$ is the density, $v$ is the speed, $L$ is the diameter, and $\eta$ is the viscosity constant. Because the Reynolds number $R e$ was $10^{-5}-10^{-4}<<1$ when $\rho=0.998 \mathrm{~g} / \mathrm{cm}^{3}, v=2-12 \mu \mathrm{m} / \mathrm{s}, L=10-22 \mu \mathrm{m}$, and $\eta=0.89 \mathrm{mPa} \cdot \mathrm{s}$, Stokes' law was applicable. Since the resistance force is estimated from the Stokes resistance $6 \pi \eta r v$, where $\eta$ is the viscosity constant, $r$ is the radius, and $v$ is the speed, it was calculated that under UV and vis illumination, the force was around 0.1-2.0 pN (Figures S14-S16). 
(a)

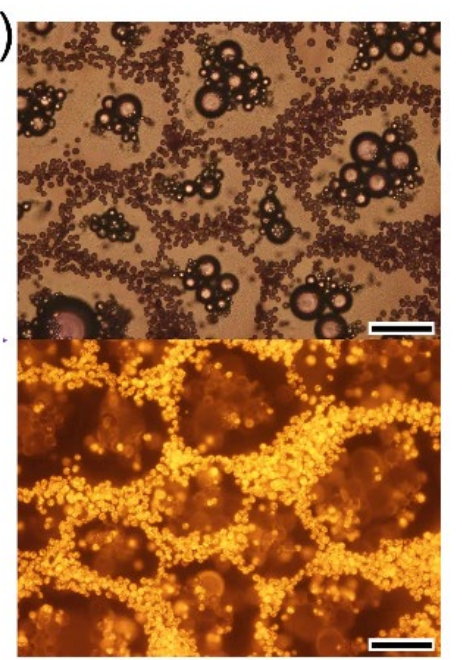

(c)

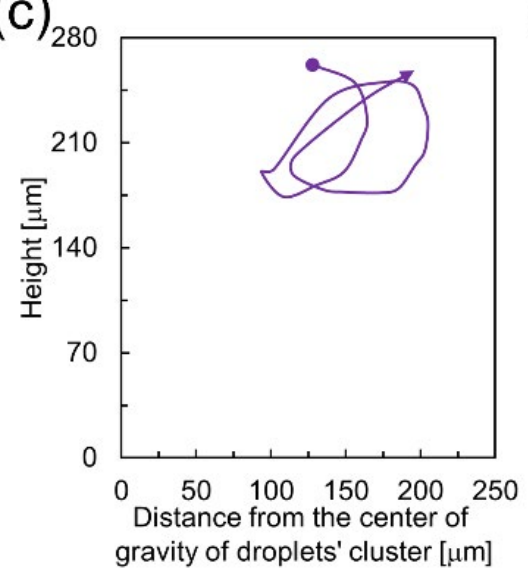

(b)

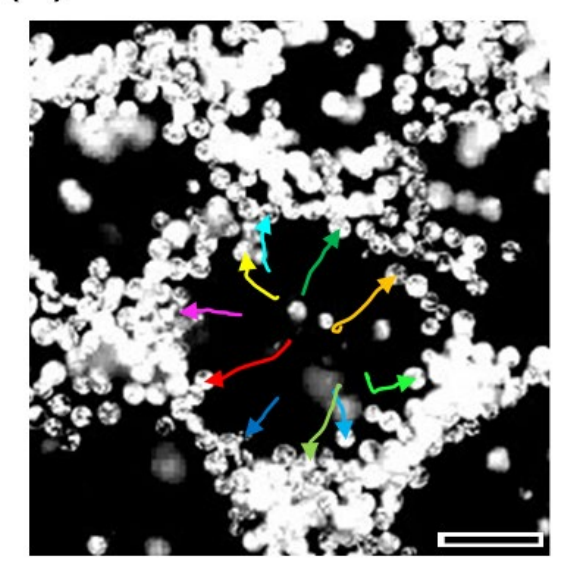

(d)

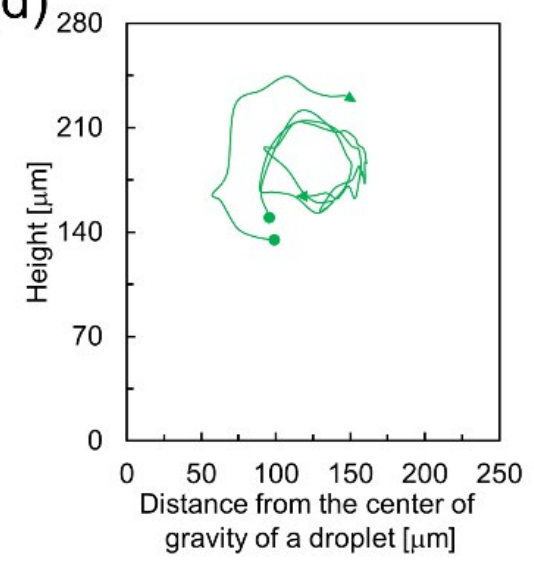

Figure 4. Visualisation of flow fields around the droplets' cluster. (a) Bright field (top) and fluorescent (bottom) images of HBA droplets in $50 \mathrm{mM}$ AzoTAB/DTAB (50/50 mol\%) surfactant solution $3 \mathrm{~min}$ after UV illumination (excitation: 530-550 nm, emission: $570-\mathrm{nm}$ ). Scale bar: $200 \mu \mathrm{m}$. (b) Trajectories of 10 fluorescence beads represented by arrows near the upper cover slip under UV illumination. These beads were accumulated in line with a circular pattern. Scale bar: $100 \mu \mathrm{m}$. (c) Trajectory of a bead moving in the $x-z$ plane under UV illumination. The circle and arrow represent the initial and end position, respectively. (d) Trajectory of a bead moving with or without circulating in the $x-z$ plane under vis illumination. Each trajectory was observed at an individual trial and superimposed. The circle and arrow represent the initial and end position, respectively. 
Because no significant difference in the flow direction was confirmed under UV and vis illumination, we investigated the influence of the absorption of light by oil on the photoisomerisation of AzoTAB at the droplet surface. At around $365 \mathrm{~nm}$, in the UV region, HBA was mostly not able to transmit UV (Figure S17). This indicates that the photoisomerisation from the trans- to cis-isomer mainly occurred at the illuminated upper side of the oil droplets (Figure 5a). On the other hand, at around $480 \mathrm{~nm}$, in the vis region, HBA almost completely transmitted light (Figure S17), indicating that the photoisomerisation from the cis- to the trans-isomer of AzoTAB occurred not only on the illuminated side but also on the opposite side of the droplet surface. The interpretation suggested that heterogeneity in the interfacial tension at the droplet surface, which is dominant for self-propelling motion, was not generated. However, this contradicts the observed results. Thus, we focused on the light refraction based on Snell's law. Since the refractive index of HBA and the surfactant solution were 1.52 and 1.33, respectively, vis light could be refracted and condensed at the lower side of the oil droplets, as if the oil droplets behaved as a lens (Figure 5b). The analysis by Snell's law suggested that the area illuminated with refracted vis light at the lower side was smaller than the area illuminated directly at the upper side, indicating that the strength of the illuminated light per unit surface area was stronger at the lower side. Thus, the interfacial tension at the lower side of the droplet became lower than that at the upper side because of faster photoisomerisation from the cis- to the trans-isomer of AzoTAB. Given that the middle area of the droplet was weakly illuminated with vis light due to light refraction, the photoisomerisation of AzOTAB occurred slowly. This indicates that the interfacial tension at the middle area was higher than at the top or bottom. In summary, Marangoni flows were considered to be induced higher from the bottom to middle than from the top to the middle of the droplet. Such quadrupole flows around each droplet likely induced the disassembly of the droplets. 
(a)

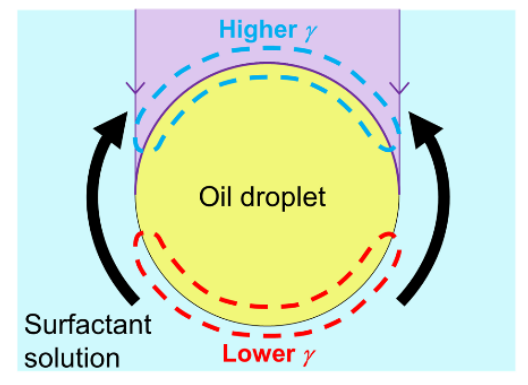

(b)

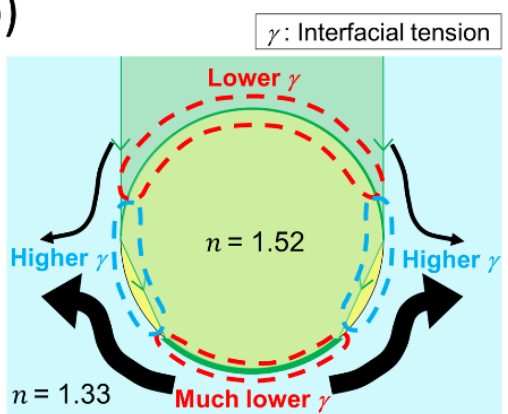

Figure 5. Schematic representations of illuminated light toward an oil droplet, thereby inducing Marangoni flows under UV illumination (a) and vis illumination (b). Italic character $n$ represents a refractive index.

Based on the flow fields induced by the photoisomerisation of AzoTAB, a mechanism for the collective behaviour of oil droplets and concurrent pattern formation was proposed. Under UV illumination, the behaviour can be classified into three stages. In the first stage, as the photoisomerisation from the trans- to the cis-isomer of AzoTAB occurred, the droplet surface of the UV-illuminated side increased the interfacial tension due to the UV absorption of the HBA droplet. Therefore, the Marangoni flows moving upwards, generated by the gradient of interfacial tension, induced the negative phototaxis of the droplet to the bottom (Figure 6a). In the second stage, droplets moved toward each other, inducing assembly (Figure 6b). There were two estimated reasons for this phenomenon. One was the hydrodynamic interaction. Convection generated around each droplet would influence the motion of droplets. The other was the gradient of the concentration of surfactants induced by the convection. The generated convection around each droplet caused the increase of the concentration of surfactants at the region between droplets, which induced the motion of each droplet toward the surfactant-rich region. Because the range of convection formed by phototactic oil droplets was limited, there was a threshold of distance at which their assembly occurred. The $\zeta$ potential of the oil droplets was positive at around $70 \mathrm{mV}$, regardless of UV illumination, indicating non-fusion of droplets. In the third stage, owing to the difference in the maximum solubilised amount between trans- and cis-AzoTAB, oil was released from the micelles, and the larger particles formed under UV illumination. The particles were swept away by convection around the droplet clusters. Because convection was induced around the droplet clusters in any direction, the particles accumulated in the shape of circles, resulting in circular 
patterns appearing around the droplet clusters. On the other hand, under vis illumination, considering the light transmittance and refraction of oil droplets, photoisomerisation from the cis- to the trans-isomer of AzoTAB proceeded. Therefore, the droplet surface at both the upper and bottom sides experienced an increase in interfacial tension, compared to the middle area. Such a gradient of interfacial tension induced Marangoni flow from the bottom to the middle and from the top to the middle of the droplets. The formed quadrupole flows around each droplet repelled each other, resulting in disassembly (Figure 6e). In addition, as photoisomerisation of AzOTAB from the cis- to the trans-isomer caused micelles to incorporate more oil, the particles that were accumulated at the patterns resolubilised into micelles, indicating the disappearance of the patterns (Figures $6 f-g$ ).

UV
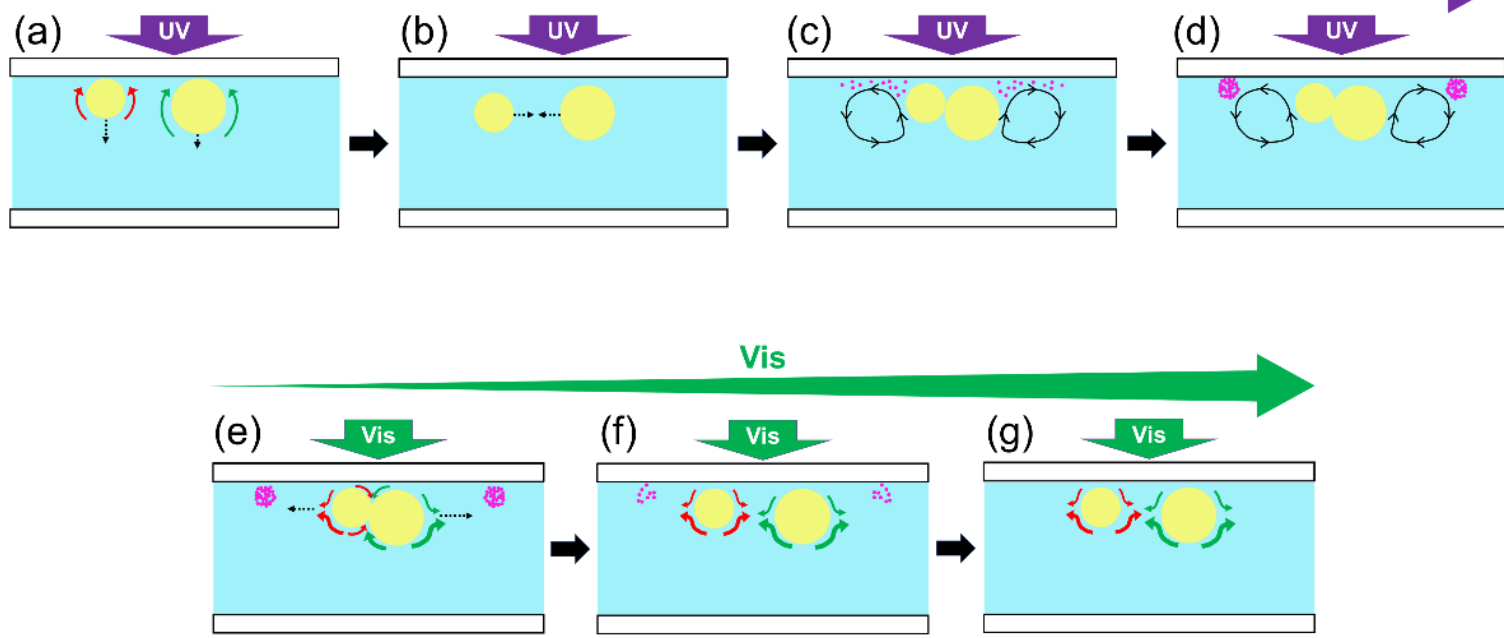

Figure 6. Schematic illustration of the proposed mechanism for collective behaviour of oil droplets and pattern formation. Under UV illumination, (a) oil droplets moved to the bottom. (b) Droplets moved toward each other, inducing assembly. (c) Oil particles (magenta) caused by solubilised oil released from micelles gradually generated due to the photoisomerisation of AzoTAB. (d) Finally, the particles were swept away by convection around droplets' clusters and accumulated at the point where the convection sank, forming circular patterns. Under vis illumination, (e) droplets repelled each other due to the formed convection around each droplet. (f) Oil particles accumulated at the patterns were incorporated into micelles and decreased. (g) The patterns that resulted from the oil particles disappeared. 


\section{Conclusion}

We have demonstrated an artificial system wherein the collective motion of multiple droplets and concurrent pattern formation emerged from the phototaxis of multiple oil droplets. By using photo-responsive azobenzene-containing surfactants, the assembly of droplets and the formation of circular patterns around the formed droplet clusters were observed under UV illumination. In addition, under subsequent vis illumination, the disassembly of droplets and disappearance of the patterns occurred. It was revealed that changes in interfacial tension were due to the photoisomerisation of AzOTAB, which induced the phototaxis of the droplets and generated characteristic convection. The convection around each droplet caused them to assemble under UV illumination, whereas they disassembled under vis illumination. In addition, a significant difference in the change in the maximum solubilised oil amount in the micelles was observed between trans- and cis-AzoTAB. This suggests the induced release of the oil component from micelles under UV illumination and the incorporation into micelles under vis illumination. Thus, the released oil components were swept away by convection and accumulated, resulting in circular patterns under UV illumination. In contrast, vis illumination resulted in the disappearance of circular patterns due to the resolubilisation of oil components into micelles. Our findings were considered to be analogous to the bioconvection of microorganisms inducing the assembly of many individuals and formation of macroscopic patterns. Therefore, this study could be a useful model for understanding the mechanism of collective motion in life in terms of physicochemical aspects.

\section{Acknowledgments}

Mr. Tsuneyoshi Torii and Ms. Mitsuyo Matsubara (Malvern Panalytical, a division of Spectris Co. Ltd.) is acknowledged for the measurements of $\zeta$ potentials. We would like to thank Editage (www.editage.com) for English language editing. This work was supported by JSPS KAKENHI Grant Number 18K05066 for T.B. 


\section{References}

[1] a) C. B. Hong, M. A. Häder, D-P. Häder, Photochem. Photobiol. 1980, 33, 373-377; b) J.

T. Pierce-Shimomura, T. M. Morse, S. R. Lockery, J. Neurosci. 1999, 19, 9557-9569.

[2] a) M. lijima, Y. E. Huang, P. Devreotes, Dev. Cell 2002, 3, 469-478; b) A. Ward, J. Liu, Z. Feng, X Z S. Xu, Nat. Neurosci. 2008, 11, 916-922.

[3] a) M. Zhao, T. Jin, C. D. McCaig, J. V. Forrester, P. N. Devreotes, J. Cell Biol. 2002, 157, 921-927; b) N. C. Sukul, N. A. Croll, J. Nematol. 1978, 10, 814-817.

[4] a) B. D. Whitaker, K. L. Poff, Exp. Cell Res. 1980, 128, 87-93; b) E. M. Hedgecock, R. L. Russell, PNAS 1975, 72, 4061-4065.

[5] M. E. H. Feinleib, G. M. Curry, Physiol. Plant 1971, 25, 346-352.

[6] D-P. Hfider, G. Colombetti, F. Lenci, M. Quaglia, Arch. Microbiol. 1981, 130, 78-82.

[7] a) N. J. Suematsu, A. Awazu, S. Izumi, S. Noda, S. Nakata, H. Nishimori, J. Phys. Soc. Jpn. 2011, 80, 064003; b) C. R. Williams, M. A. Bees, J. Exp. Biol. 2011, 214, 2398-2408.

[8] a) P. J. M. Van Haastert, P. N. Devreotes, Nat. Rev. Mol. Cell Biol. 2004, 5, 626-634; b) C. I. Bargmann, Chemosensation in C. elegans (October 25, 2006), WormBook, ed. The C. elegans Research Community, WormBook, 2006. c) O. A. Sineshchekov, K. Jung, J. L. Spudich, PNAS 2002, 99, 8689-8694.

[9] N. J. Suematsu, S. Nakata, Chem. Eur. J. 2018, 24, 6308-6324.

[10] a) Y. Tu, F. Peng, J. M. Heuvelmans, S. Liu, R. J. M. Nolte, D. A. Wilson, Angew. Chem. Int. Ed. 2019, 58, 8687-8691; Angew. Chem. 2019, 131, 8779-8783; b) E. Uchida, R. Azumi, Y. Norikane, Nat. Commun. 2015, 6, 7310; c) S. Nakata, T. Miyaji, Y. Matsuda, M. Yoshii, M. Abe, Langmuir 2014, 30, 7353-7357; d) T. Ikegami, Y. Kageyama, K. Obara, S. Takeda, Angew. Chem. Int. Ed. 2016, 55, 8239-8243; Angew. Chem. 2016, 128, 8379-8383; e) H. Wang, M. Pumera, Chem. Rev. 2015, 115, 8704-8735.

[11] C. Chen, F. Mou, L. Xu, S. Wang, J. Guan, Z. Feng, Q. Wang, L. Kong, W. Li, J. Wang, Q. Zhang, Adv. Mater. 2017, 29, 1603374.

[12] S. Gangwal, O. J. Cayre, M. Z. Bazant, O. D. Velev, Phys. Rev. Lett. 2008, 100, 058302. [13] W. F. Paxton, K. C. Kistler, C. C. Olmeda, A. Sen, S. K. St. Angelo, Y, Cao, T. E. Mallouk, P. E. Lammert, V. H. Crespi, J. Am. Chem. Soc. 2004, 126, 13424-13431.

[14] M. Xuan, Z. Wu, J. Shao, L. Dai, T. Si, Q. He, J. Am. Chem. Soc. 2016, 138, 6492-6497. [15] a) A. Diguet, R. Guillermic, N. Magome, A. Saint-Jalmes, Y. Chen, K. Yoshikawa, D. Baigl, Angew. Chem. Int. Ed. 2009, 48, 9281-9284; Angew. Chem. 2009, 121, 9445-9448; b) L. Florea, K. Wagner, P. Wagner, G. G. Wallace, F. Benito-Lopez, D. L. Officer, D. Diamond, Adv. Mater. 2014, 26, 7339-7345; c) K. Suzuki, T. Sugawara, ChemPhysChem 2016, 17, 2300-2303; d) S. Kaneko, K. Asakura, T. Banno, Chem. Commun. 2017, 53, 2237-2240. 
[16] M. Ichikawa, F. Takabatake, K. Miura, Phys. Rev. E 2013, 88, 012403.

[17] a) M. M. Hanczyc, T. Toyota, T. Ikegami, N. Packard, T. Sugawara, J. Am. Chem. Soc. 2007, 129, 9386-9391; b) I. Lagzi, S. Soh, P. J. Wesson, K. P. Browne, B. A. Grzybowski, J. Am. Chem. Soc. 2010, 132, 1198-1199; c) S. Miura, T. Banno, T. Tonooka, T. Osaki, S. Takeuchi, T. Toyota, Langmuir 2014, 30, 7977-7985.

[18] J. Čejková, M. Novák, F. Štěpánek, M. M. Hanczyc, Langmuir 2014, 30, 11397-11944.

[19] a) T. Ban, K. Tani, H. Nakata, Y. Okano, Soft Matter 2014, 10, 6316-6320; b) T. Ban, H. Nakata, J. Phys. Chem. B 2015, 119, 7100-7105.

[20] S. Yeh, M. Seul, B. I. Shraiman, Nature 1997, 386, 57-59.

[21] M. Ibele, T. E. Mallouk, A. Sen, Angew. Chem. Int. Ed. 2009, 48, 3308-3312; Angew. Chem. 2009, 121, 3358-3362.

[22] a) S. Thutupalli, R. Seemann, S. Herminghaus, New J. Phys. 2011, 13, 073021; b) S. Thutupalli, D. Geyer, R. Singh, R. Adhikari, H. A. Stone, PNAS 2018, 115, 5403-5408; c) S. Tanaka, S. Nakata, T. Kano, J. Phys. Soc. Jpn. 2017, 86, 101004; d) J. Čejková, K. Schwarzenberger, K. Eckert, S. Tanaka, Colloids Surf. A 2019, 566, 141-147; e) C. Krüger, C. Bahr, S. Herminghaus, C. C. Maass, Eur. Phys. J. E 2016, 39, 64.

[23] a) N. Yoshinaga, K. H. Nagai, Y. Sumino, H. Kitahata, Phys. Rev. E 2012, 86, 016108; b) Y. Kasuo, H. Kitahata, Y. Koyano, M. Takinoue, K. Asakura, T. Banno, Langmuir 2019, 35, $13351-13355$. 